Density for Fermions in a Harmonic-Oscillator Potential

This article has been downloaded from IOPscience. Please scroll down to see the full text article. 1986 Europhys. Lett. 1545

(http://iopscience.iop.org/0295-5075/1/11/001)

View the table of contents for this issue, or go to the journal homepage for more

Download details:

IP Address: 139.165.107.21

The article was downloaded on 05/12/2011 at 15:52

Please note that terms and conditions apply. 
Europhys. Lett., 1 (11), pp. 545-548 (1986)

\title{
Density for Fermions in a Harmonic-Oscillator Potential.
}

\author{
J. CUGNON \\ Université de Liège, Physique Nucléaire Théorique, \\ Institut de Physique au Sart Tilman, B.5 - B-4000 Liège 1 (Belgique) \\ O. HAROUNA \\ Université de Niamey, Service de Physique, \\ B.P. 10662 Niamey (République du Niger)
}

(received 3 February 1986; accepted 25 March 1986)

PACS. 21.10. - General and average properties of nuclei; properties of nuclear energy levels.

\begin{abstract}
We show explicitly that the ground-state density of a system of noninteracting fermions in a harmonic-oscillator potential well can be given in terms of the wave functions of the single-particle states around the Fermi level.
\end{abstract}

In a previous publication [1], we demonstrated that the derivative of the density for a system of noninteracting fermions occupying the lowest levels in a three-dimensional harmonic-oscillator potential can be written in terms of a few radial wave functions. Typically, if $N$ major oscillator shells are occupied, the derivative implies only the last occupied shell and the first nonoccupied one. Here, we demonstrate a similar property for the density itself.

That such a relation should hold is rather obvious. Indeed the density (for a spin- $\frac{1}{2}$ fermions) is given by

$$
p(r)=\frac{1}{2 \pi} \sum_{\{n, l\} \leqslant F}(2 l+1) R_{n l}(r) R_{n l}(r)
$$

where $R_{n l}$ is the normalized radial wave function for the state $\{n, l\}$. The summation runs up to the last occupied major shell denoted by $F$, such that $2 n+l \leqslant F$. There exists a relationship [2] linking $R_{n, l}, R_{n, l+1}$ and $R_{n+1, l-1}$ (see below). Successive use of this relation in eq. (1) would give the desired result. However, this direct procedure is rather involved and many manipulations are needed to reach the simple result that we exhibit below.

Here, we produce a simple demonstration using our previous result. We take the derivative of eq. (1) (where we put the oscillator parameter $a=\sqrt{\hbar / m \omega}$ equal to one), and use the expression for the derivative of $R_{n l}[2,3]$. We get

$$
\frac{\mathrm{d} \rho}{\mathrm{d} r}=\frac{1}{\pi} \sum_{n, l \leqslant F}(2 l+1) \frac{R_{n l}(r)}{r}\left\{-R_{n l}+\left(2 n+l+1-r^{2}\right) R_{n l}-2\left[n\left(n+l+\frac{1}{2}\right)\right]^{\frac{1}{2}} R_{n-1, l}\right\}
$$


or

$\frac{\mathrm{d} \rho}{\mathrm{d} r}=-\frac{3}{r} f+\frac{1}{\pi} \sum_{n, l \leqslant F}(2 l+1) \frac{R_{n l}}{r}\left\{\left(2 n+l+\frac{3}{2}-r^{2}\right) R_{n l}-2\left[n\left(n+l+\frac{1}{2}\right)\right]^{\frac{1}{2}} R_{n-1 . l}\right\}$

with the help of the recurrence relation [2]

$\left[n\left(n+l+\frac{1}{2}\right)\right]^{\frac{1}{2}} R_{n-1, l}-\left(2 n+l+\frac{3}{2}-r^{2}\right) R_{n l}+\left[(n+1)\left(n+l+\frac{3}{2}\right)\right]^{\frac{1}{2}} R_{n+1, l}=0$,

eq. (3) becomes

$\frac{\mathrm{d} \rho}{\mathrm{d} r}=-\frac{3}{r} \rho+\frac{1}{\pi} \sum_{n, l \leqslant F}(2 l+1) \frac{R_{n l}}{r}\left\{\left[(n+1)\left(n+l+\frac{3}{2}\right)\right]^{\frac{1}{2}} R_{n+1, l}-\left[n\left(n+l+\frac{1}{2}\right)\right]^{\frac{1}{2}} R_{n-1, l}\right\}$.

This can be rewritten as

$$
\frac{\mathrm{d} \rho}{\mathrm{d} r}=-\frac{3}{r} \rho+\frac{1}{\pi} \sum_{\{n, l\}=F, F-1}(2 l+1)\left[(n+1)\left(n+l+\frac{3}{2}\right)\right]^{\frac{1}{2}} \frac{R_{n l} R_{n+1, l}}{r},
$$

where the summation is restricted to the last two occupied major shells. Using the relation

$$
\left(n+l+\frac{3}{2}\right)^{\frac{1}{2}} R_{n l}=r R_{n, l+1}+(n+1)^{\frac{1}{2}} R_{n+1, l},
$$

the summation on the major shell $F-1$ can be put in the form

$$
\sum_{\{n, l\}=F-1}(2 l+1) \frac{R_{n+1, l}}{r}(n+1)^{\frac{1}{2}}\left[r R_{n, l+1}+(n+1)^{\frac{1}{2}} R_{n+1, l}\right]
$$

or

$$
\sum_{\{n, l\}=F}\left(1-\delta_{l 0}\right)(2 l-1) \frac{1}{r} R_{n+1, l-1}(n+1)^{\frac{1}{2}}\left[r R_{n, l}+(n+1)^{\frac{1}{2}} R_{n+1, l-1}\right] .
$$

Relation (7) can also be used to transform the term corresponding to $F$ in eq. (6). Gathering all the results, we obtain

$$
\begin{aligned}
& \frac{\mathrm{d} \rho}{\mathrm{d} r}=-\frac{3}{r} p-\frac{1}{\pi} \sum_{n, l=F}\left\{(2 l+1)\left(n+l+\frac{3}{2}\right)^{\frac{1}{2}} R_{n l} R_{n, l+1}-(2 l+1)\left(n+l+\frac{3}{2}\right) \frac{R_{n l}^{2}}{r}-\right. \\
& \left.-\left(1-\delta_{l 0}\right)(2 l-1)(n+1)^{\frac{1}{2}} R_{n l} R_{n+1, l-1}-\left(1-\delta_{l 0}\right)(2 l-1)(n+1) \quad \frac{\left(R_{n+1, l-1}\right)^{2}}{r}\right\} .
\end{aligned}
$$

Using our previous result [1], we finally get

$$
\begin{aligned}
f(r)=\frac{1}{3 \pi} & \sum_{\{n, l\}=F}\left\{-l\left(n+l+\frac{3}{2}\right)^{\frac{1}{2}} r R_{n l} R_{n, l+1}+(2 l+1)\left(n+l+\frac{3}{2}\right) R_{n l}^{2}+\right. \\
& \left.+\left(1-\delta_{l 0}\right)(l-1)(n+1)^{\frac{1}{2}} r R_{n l} R_{n+1, l-1}+\left(1-\delta_{l 0}\right)(2 l-1)(n+1) R_{n+1, l-1}^{2}\right\} .
\end{aligned}
$$

As announced, this expression involves the wave functions of the last occupied shell and of the first unoccupied one only. 
As for $\mathrm{d} \rho / \mathrm{d} r$, expression (11) could be obtained from one of the many sum rules which have been popularized in nuclear physics [4-7]. In this particular case, the sum rule is the following:

$$
-\frac{i \hbar}{2 m} 3_{i}(\boldsymbol{r})=\sum_{n}\langle 0|\boldsymbol{j}(\boldsymbol{r})| n\rangle \cdot\langle n|\hat{\boldsymbol{r}}| 0\rangle
$$

where

$$
\hat{\boldsymbol{r}}=\sum_{k} \boldsymbol{r}^{(k)}, \quad \boldsymbol{j}=\frac{1}{2 m} \sum_{k}\left\{\boldsymbol{p}^{(k)}, \delta\left(\boldsymbol{r}-\boldsymbol{r}^{k}\right)\right\} .
$$

Here $k$ labels the particles (of mass $m$ ) and the curly brackets indicate the anticommutator. In eq. (12), $|0\rangle$ denotes the ground state of the system and $|n\rangle$ is any possible state. In the case of the harmonic-oscillator Hamiltonian, $\hat{r}$ has nonvanishing matrix element when $|n\rangle$ is a one particle-one hole state with the hole in the last occupied major shell and the particle in the first unoccupied major shell. Therefore, the property announced in the beginning could be obtained this way. However, to work out the final expression (11) requires cumbersome calculations involving repeated use of Clebsch-Gordan algebra and of relations fulfilled by products of spherical harmonics.

As discussed in ref. [1], expression (11) involves more than a curious property of the harmonic-oscillator wave functions and can be of interest in at least three cases:

1) A system of particles interacting via harmonic-oscillator potential can be reduced, to some extent, to a system of independent particles in a potential well [8].

2) The property (11) is approximately valid for a more realistic potential like a WoodsSaxon potential. The property followed by $\mathrm{d}_{\mathrm{o}} / \mathrm{d} r$ demonstrated in ref. [1] is also approximately valid, as we showed explicitly. The only practical point is the determination of a length parameter. We observed in ref. [1] that using $a_{0}=\left[\hbar^{2} / 2 m\left(\bar{\varepsilon}_{1}-\bar{\varepsilon}_{2}\right)\right]^{\frac{1}{2}}$, where $\bar{\varepsilon}_{1(2)}$ is the average single-particle energy in the lowest unoccupied (last occupied) shell, provides the best choice.

3) As observed first by LiU and BRown [9], a good calculation (by standard techniques, like RPA e.g.) of the properties of the collective excitations of a nuclear system requires a good single-particle spectrum near the Fermi level. The property we derived in ref. [1] in fact relates this part of the spectrum (and the wave functions) to the quantity $\mathrm{d} \rho / \mathrm{d} r$, which is responsible of the collective excitations, corroborating in some sense the observation of ref. [9]. Property (11) is interesting in the same respect, since some collective excitations are due to operators proportional to the density or more generally to a linear combination of the density and its derivative (see f.i. ref. [10]).

\section{REFERENCES}

[1] J. Cugnon and O. HaRouna: Lett. Nuovo Cimento, 36, 279 (1983).

[2] M. Moshinsky: The Harmonic Oscillator in Modern Physics: From Atom to Quarks (Gordon and Breach, London, 1969).

[3] M. Abramowitz and I. A. Stegun: Handbook of Mathematical Functions (Dover Publ., New York, N. Y., 1972).

[4] J. V. Noble: Ann. Phys. (N. Y.), 67, 98 (1971); Phys. Rep. C, 40, 241 (1978). 
[5] S. FAllienos: Proceedings of the International Conference on Nuclear Structure Studies using Electron Scattering and Photoreactions, edited by K. SHODA and H. Ur (Tohoku Univ., Sendai, 1972), p. 185.

[6] H. Ui and T. Tsukamoto: Prog. Theor. Phys., 51, 1377 (1974).

[7] T. SuzukI and D. T. Rowe: Nucl. Phys. A, 286, 307 (1977).

[8] D. J. THouless: The Quantum Mechanics of Many Body Systems, 2nd edition (Academic Press, New York, N. Y., 1972).

[9] K. F. LIU and G. E. Brown: Nucl. Phys., 265, 385 (1986).

[10] H. SAGAWA and N. VAN GiaI: Phys. Lett. B, 127, 393 (1983). 